\title{
ENERGY-DISPERSIVE X-RAY SPECTRUM SIMULATION AND EMPRICAL OBSERVATION OF 22NM NODE HIGH-K METAL GATE STRUCTURE
}

\author{
Imen Rezadad ${ }^{1,2}$, Brenda Prenitzer ${ }^{2}$, Stephen Schwarz $^{2}$, Brian Kempshall ${ }^{2}$, Robert Peale ${ }^{1}$
}

1. Physics Department, University of Central Florida, Orlando, Florida, USA.

2. NanoSpective, Inc., Orlando, Florida, USA.

Characterization of advanced technology node integrated circuits is an ever-increasing challenge. In order for analytical capabilities to keep pace with technology it is important to carefully consider all aspects in the design and execution of experiments. It is essential to be able to distinguish artifact from meaningful data and to recognize the limitations as well as the capabilities of a given technique. Some critical considerations include specimen preparation, physical limitations of the instrumentation, alignment and calibration as well as user selected acquisition parameters. The effects of sampling size, signal to noise ratio, specimen geometry, instrumentation and accelerating voltage on the microanalysis of multilayered metal gate structures created using atomic layer deposition (ALD) are presented.

Empirical results are compared with modeled data for energy dispersive spectroscopy (EDS) line profiles through two different ALD thin film stacks routinely used in high-k metal gates (HKMG) at the $22 \mathrm{~nm}$ node. The question of whether and under what conditions do EDS line profiles have adequate lateral spatial resolution to sufficiently differentiate diffusion at monolayer length scales is investigated. Analytical calculations using the Jones matrix formalism showing the degree of beam spreading will be presented for two different TEM configurations. [1] Electron trajectories through the specimen thickness are also simulated using Casino.[2] EDS simulations are done using MC X-Ray [3] to represent the compositional analysis of two different thin film stacks containing slices of $\mathrm{Si}, \mathrm{TiN}, \mathrm{HfO}_{2}$ and $\mathrm{SiO}_{2}$ and TiAl, TiNTa, Ta, TiN, $\mathrm{HfO}_{2}, \mathrm{SiO}_{2}$ and $\mathrm{Si}$. Simulation results are correlated with empirical measurements performed on gates extracted from two different devices. Spectra from analyses performed on an FEI Tecnai F30 TEM with an EDAX Sapphire Si(Li) EDS detector will be contrasted with spectra obtained on a probe corrected FEI Titan 80-300 using a Bruker SuperX4 silicon drift detector (SDD) EDS system. Figure 1 shows an example of an analysis performed on the Titan.

The simulations are performed on atomically smooth interfaces to illustrate the profound effect that user defined parameters can have on data acquisition and interpretation. Figure 2 shows the effect that undersampling can have on the shape of a line profile. A region may be undersampled if the step size is too large with respect to the feature size. A similar loss in lateral spatial resolution will be observed even if the step size is small enough but the probe diameter is too large with respect to the feature size. Figure 4 shows the effect that probe position can have on the shape of a line profile. The apparent location of the interface can be displaced by a distance as large as the step size. Similar to undersampling, the effects of a misplaced sampling probe can be minimized by selecting the appropriate step size with respect to the feature of interest and the resolution requirements for your analysis.

\section{References}

[1] David B. Williams, “Transmission Electron Microscopy”, (Springer, New York), P. 665.

[2] D. Drouin et al., Scanning 29 (2007), 92-101.

[3] http://montecarlomodeling.mcgill.ca/ 


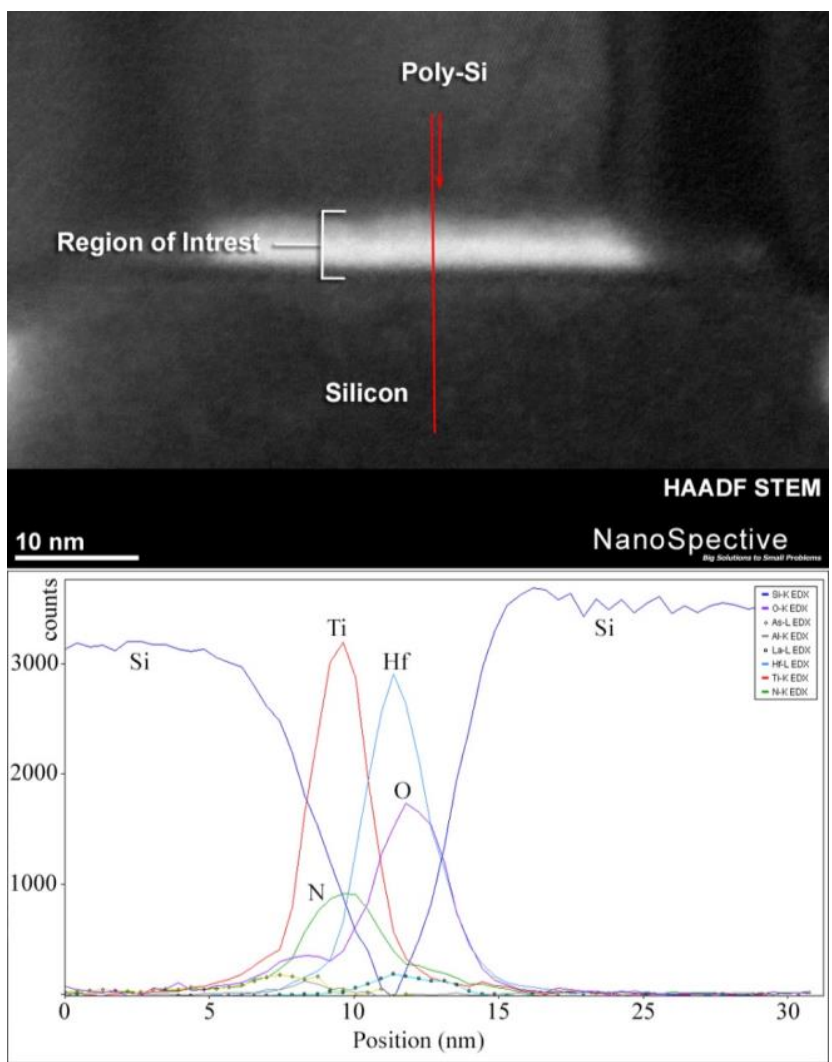

Fig. 1. STEM image and EDS line profile acquired with adequate sampling through the HKMG stack of an NMOS transistor in an advanced technology node device

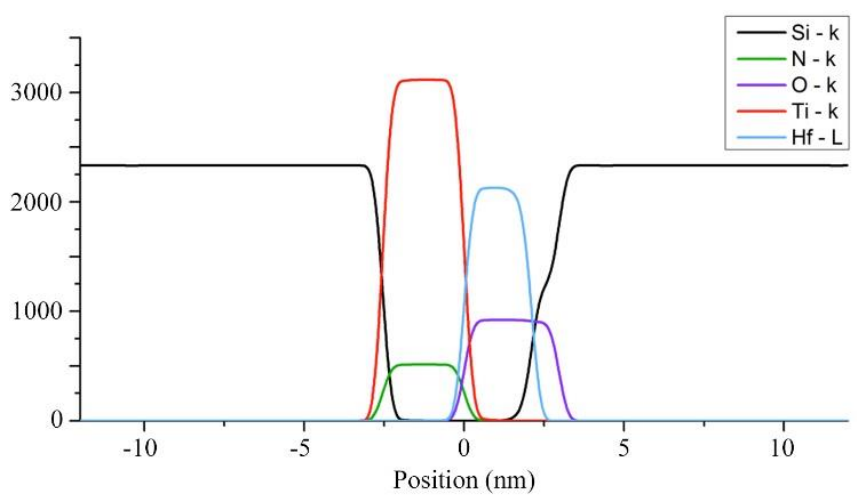

Fig. 3. Simulated EDS spectrum for a structure and sampling corresponding to that shown in figure 1. Compared to the empirical spectrum, the interfaces show steeper slopes suggesting that the profiles observed during the experiment are attributable to actual diffusion.

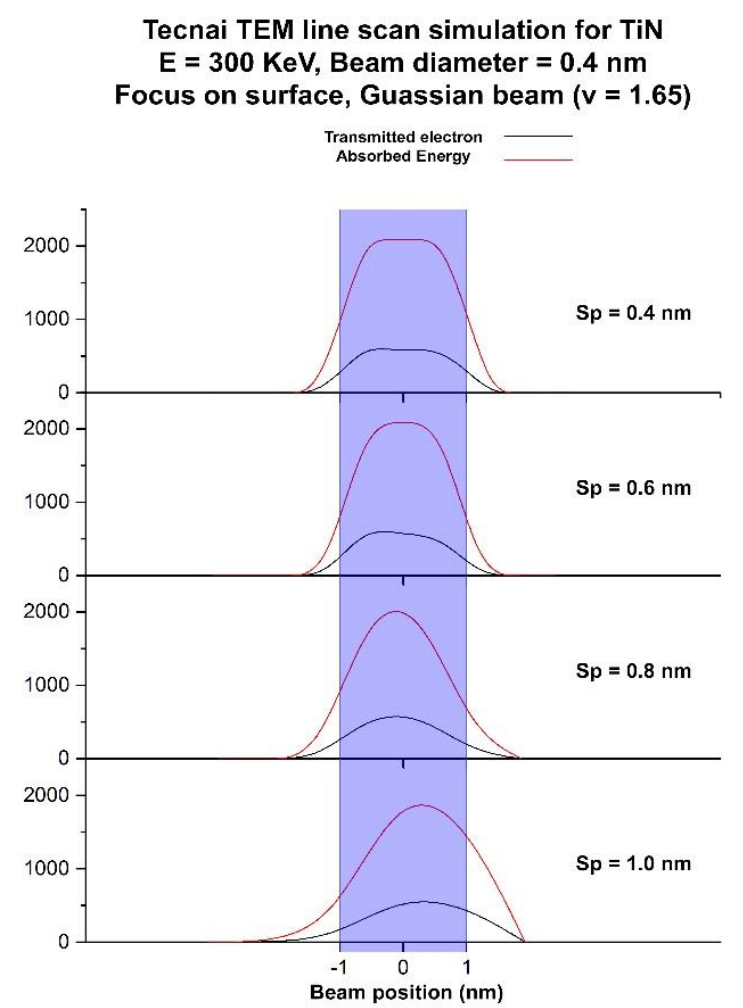

Fig. 2. The effect of undersampling on profile shape. The skewed shape erroneously resembles a diffusion profile.

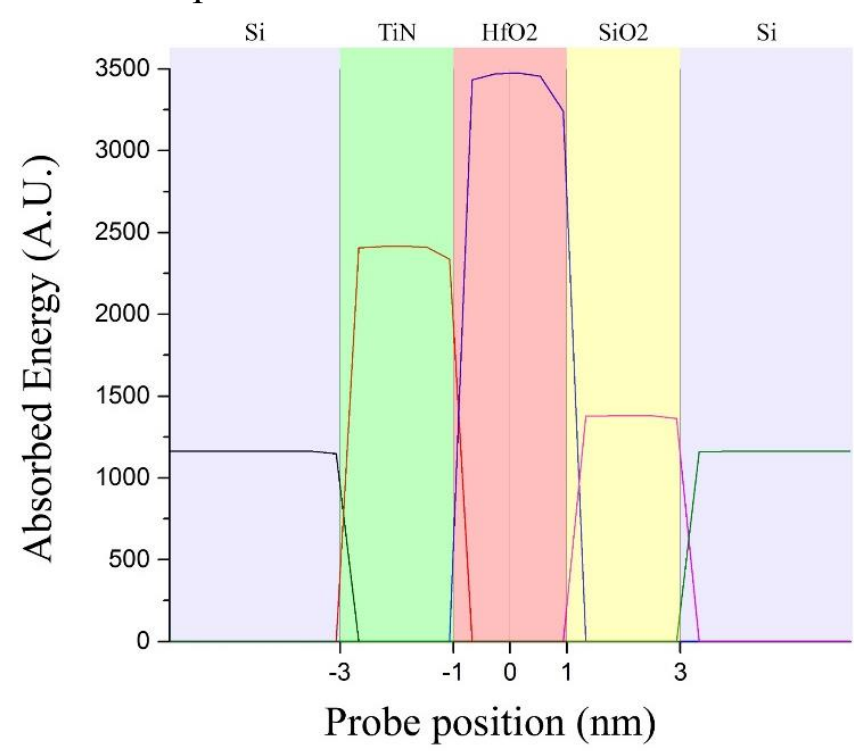

Fig. 4. The effect of probe position with respect to interface. The apparent position of the interface can be displaced by a distance as large as the step size. 